\title{
Design of Modified Tabu Search (MTS) Algorithm, an Optimization Technique for Intelligent Routing of an IOT Network with an aim to Improving the Efficiency
}

\section{Anukriti Sharma ( $\nabla$ satvansh2015@gmail.com )}

Maharishi Markandeshwar Group of Institutions: Maharishi Markandeshwar University

Sharad Sharma

Maharishi Markandeshwar (Deemed to be) University

Dushyant Gupta

Institute of Hons. and evening studies, kurukshetra university

\section{Research Article}

Keywords: Modified Tabu Search, Tabu search, Distance to previous node, Distance to Zero, Base network algorithm, Shortest path

Posted Date: August 19th, 2021

DOl: https://doi.org/10.21203/rs.3.rs-554510/v1

License: (c) (1) This work is licensed under a Creative Commons Attribution 4.0 International License.

Read Full License 


\title{
Design of Modified Tabu Search (MTS) Algorithm, an Optimization Technique for Intelligent Routing of an IOT Network with an aim to Improving the Efficiency
}

\author{
Anukriti Sharma ${ }^{*}$, Sharad Sharma ${ }^{2}$, Dushyant Gupta ${ }^{3}$ \\ ${ }^{1 *}$ Electronics and Communication Engineering Department, Maharishi Markandeshwar \\ (deemed to be University), Mullana-Ambala, Haryana, India \\ ${ }^{2}$ Electronics and Communication Engineering Department, Maharishi Markandeshwar \\ (deemed to be University), Mullana- Ambala, Haryana, India \\ ${ }^{3}$ Electronics Department, Institute of Honors \& Evening Studies, Kurukshetra University, \\ Kurukshetra, Haryana, India
}

E-mail address: satvansh2015@gmail.com ${ }^{1 *}$, sharadpr123@rediff.com ${ }^{2}$, gupty2kuk@yahoo.co.uk ${ }^{3}$

\begin{abstract}
In this study, Modified Tabu Search (MTS) optimization technique with metaheuristic approach has been proposed. This technique is based on an example of salesman travelling problem that could be resolved effectively using metaheuristic Tabu Search algorithms where implementation of an intelligent routing technique has been proposed in order to improving the efficiency of an IOT network. Further, in this piece of research work, existing routing techniques have been studied to predict a cost-effective shortest path for different network size. It was observed that Modified Tabu Search (MTS) is quite an efficient technique in surpassing all existing routing techniques, and therefore, could prove to be one of the most preferable technique in a real time scenario to be applied to an IOT based network.
\end{abstract}

Keywords: Modified Tabu Search; Tabu search; Distance to previous node; Distance to Zero; Base network algorithm; Shortest path. 


\section{Introduction}

Tabu Search is defined as a meta heuristic search approach that has been overlaid on another heuristic approach for the purpose of finding the optimum solution [1]. In Tabu Search, cycling of events are prohibited with the use of Tabu list. Tabu list records the recent search events and avoids solution to move in prior visited solution space [2]. This approach permits Tabu search to competently recognize high-quality solutions. Further, Tabu search is capable to search solutions related to operational research and artificial intelligence problems. Unlike, Simulated Annealing and Genetic Algorithm, Tabu search make astute use of previous search to affect its future [3]. Additionally, Tabu search provides substitutes for neural network memory, making it possible to develop neural network operations in realistic scenario $[4,5]$.

A lot of studies have recently been performed to enhance the TS algorithm efficiency. Such studies concentrate in particular on mitigating the common drawbacks. Some studies make use or modify the existing algorithm for the purpose of enhancing the speed when it comes to storage [6]. It has been observed by various researchers that Tabu Search approach and its derivatives offer solutions that closely resemble to an optimum solution for complex problems. There are several problems in the natural world that are complex, apart from being having different constraints. Optimization methods are, therefore, mandatory to have quick search mechanism and a high level of adaptability [7].

I. Mathlouthi et al. [8] proposed Tabu search methodology for solving problem related to technician scheduling and routing where the results have been reported by considering a task with 200 size of nodes. Alian Hertz et al. [9] implemented Tabu search algorithm for finding optimal solution for planar networks and the results so computed revealed that solution computation time is very less in case of Tabu search. M. Chen et al. [10] implemented a novel Tabu search technique for finding optimal solution for university course timetable problem. Sharad Sharma et al. [11-14] proposed different routing approaches namely Ant Colony Optimization, firefly, Big Bang Big Crunch using fuzzy logic with the aim to find the shortest ILC (integrated link cost) path within a given time to highlight that proposed approaches efficiently reduces the network complexity.

The proposed research work of designing an algorithm, being named as Modified Tabu Search (MTS), has been carried out by keeping in mind various constraints that has already been explored by various researchers. In this paper, the manuscript has been organized into four parts for the sake of clarity. In first part, the proposed routing technique being named as 
Modified Tabu Search (MTS) depicts an explanation on the process of computation of the shortest distance among node counts verses least cost function. Second section corroborates the generated pseudo-code for the Modified Tabu Search (MTS) algorithm while third part covers the developed algorithm that has been applied in dynamic scenarios. Finally, in the last section effectiveness of the proposed algorithm has been measured by comparing it with existing routing techniques.

\section{Modified Tabu Search (MTS) Algorithm}

Modified Tabu Search (MTS) is an extension of Tabu Search and considered as metaheuristic and global optimization algorithm. In MTS, search has been done locally as in case of Tabu Search. The objective of Modified Tabu Search (MTS) is to minimize the number of cycles generated during implementation of local search technique by explicitly monitoring the search and countering the occurrence of cycles and their repetition by using Tabu tenure, Tabu list size. Modified Tabu Search (MTS) technique keeps on searching as long as the least cost integral function with respect to distance between nodes is achieved, thereby, giving improved results in comparison with the conventional Tabu Search.

Pseudocode for the Modified Tabu Search algorithm has been provided by algorithms reported in existing literature for the purpose of minimizing the cost function. The 'Algorithm A' that described the Pseudocode for Modified Tabu Search (MTS) is based on Improved Tabu Search (ITS) that has already been proposed by Battiti and Tecchiolli [15]. The procedure has been modified for brevity to exude the diversification procedure escape move. Further, 'Algorithm B' described the memory-based reaction that manipulates the size of the prohibition period in response to identified cycles in the ongoing search. 'Algorithm C' described the selection of the best move from a list of candidate moves in the neighbourhood of a given solution. The function permits prohibited moves in the case where a prohibited move is better than the best-known solution and the selected admissible move called 'Aspiration'. 'Algorithm D' determined whether a given neighbourhood move is Tabu based on the current prohibition period and has been employed by sub-functions of the algorithm function.

\footnotetext{
Algorithm A: Pseudocode for Modified Tabu Search

Input: Iteration max, Increase, Decrease, Problem Size

Output: Sbest

1 Scurr $\leftarrow$ Construct Initial Solution ();
} 


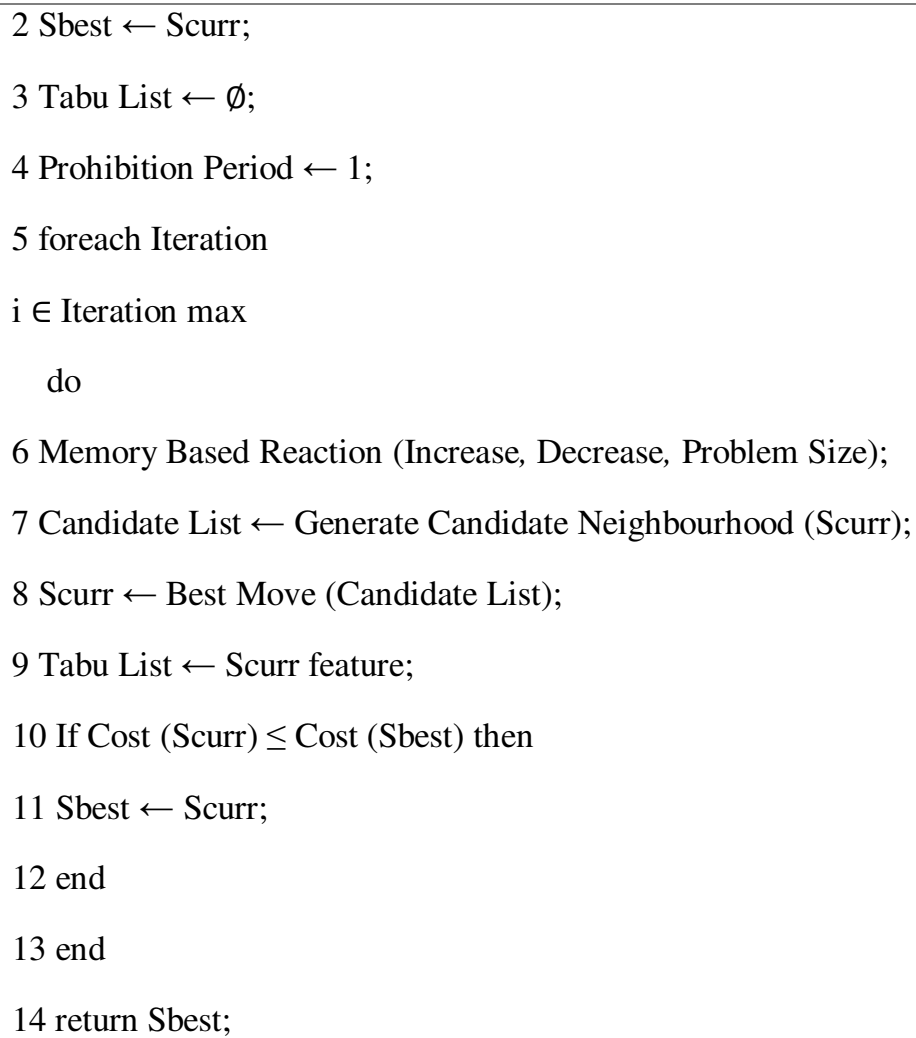

The steps involved in the MTS pseudo code are as follows.

Step 1: Forming the Tabu list: At the beginning of MTS, the initial network consists of $n$ number of nodes. Further, the first node is created from the output. Then, $n-1$ nodes are made from the solution. The prohibition period (time each iteration will take) is fixed.

Step 2: Forming a Neighbourhood setting: Each iteration, $i$ belong to maximum iterations. All neighbours generated from the current solution are placed on the candidate list. The best value out of the candidate list is passed to the current plan.

Step 3: Updating solution and current list: The current list consists of the best available neighbour. The Tabu list is also updated with the best solution.

Step 4: Repeat step 2-3 until the best solution is obtained.

\section{Algorithm B: Pseudocode for the Memory Based Reaction function}

Input: Increase, Decrease, Problem Size

Output:

1 if Have Visited Solution Before (Scurr, Visited Solutions) then

2 Scurrt $\leftarrow$ Retrieve Last Time Visited (Visited Solutions, Scurr); 


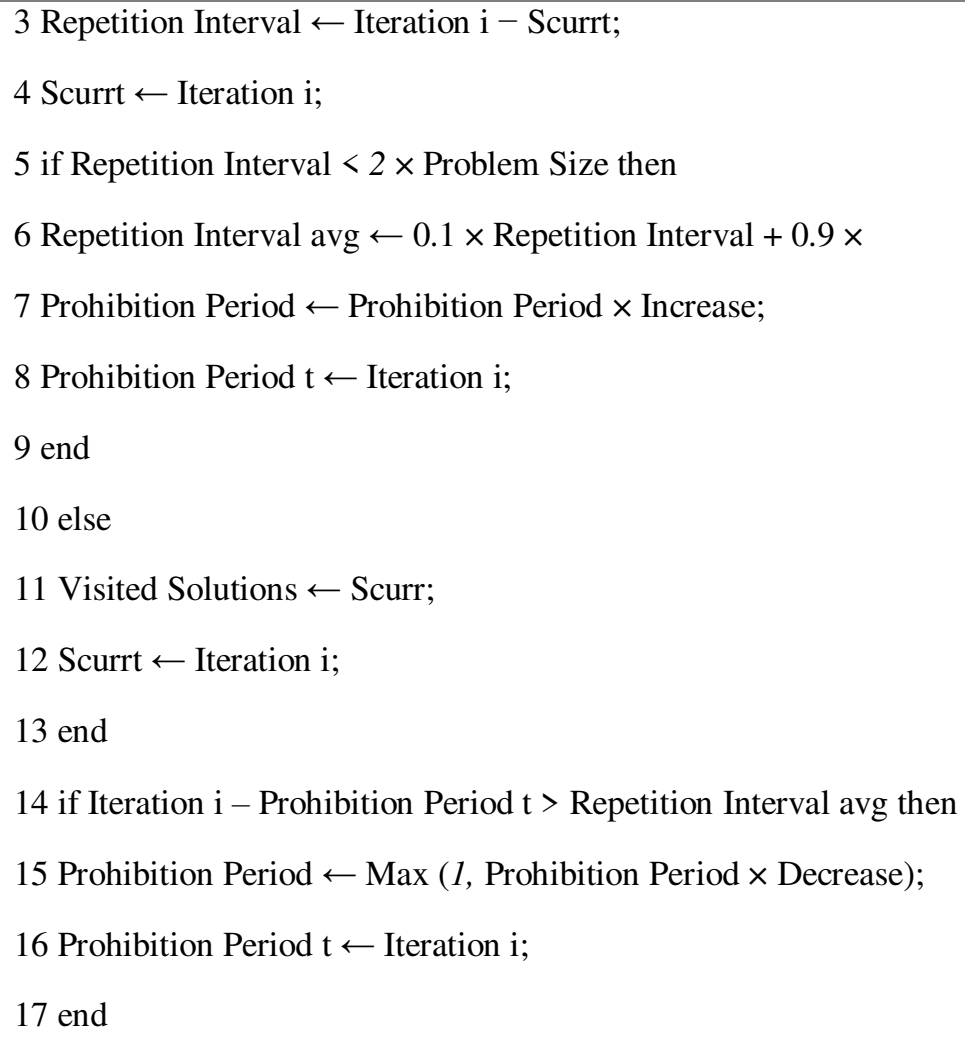

The steps involved in Memory-based reaction function pseudo code are as explained below:

Step 1: After the declaration of three input parameters, as shown in the algorithm. In the case of visiting any node-whether current or previous visit. The number of repetitions occurs according to the number of iterations in the current value. If no occurrence occurred, current list will remain same.

Step 2: In case the condition for an interval of repetition is less than twice the problem size, that is considered as the distance from one to another.

Step 3: Reducing time for maximum iteration is the main objective of keeping it weightless. The condition for increases in iterations is limited to the prohibition period, because repetition period cannot be reduced.

\section{Algorithm C: Pseudocode for the Best Move function}

Input: Problem size

Output: Scurr

1Candidate List admissible $1 \leftarrow$ Get Admissible Moves (Candidate List); 
2 Candidate List tabu $\leftarrow$ Candidate List - Candidate List admissible;

3 if Size (Candidate List admissible) $<2$ then

4 Prohibition Period $\leftarrow$ Problem Size -2 ;

5 Prohibition Period $t \leftarrow$ Iteration $\mathrm{i}$;

6 end

7 Scurr $\leftarrow$ Get Best(Candidate List admissible);

8 Sbest tabu $\leftarrow$ Get Best (Candidate List tabu);

9 if Cost (Sbest tabu) < Cost (Sbest) $\wedge$ Cost (Sbest tabu) < Cost (Scurr)

then

10 Scurr $\leftarrow$ Sbest tabu;

11 end

12 return Scurr;

The steps involved in the best move function pseudo code are as described.

Step 1: This algorithm reduces problem size. The candidate list includes variable, where weight is inserted for each node.

Step 2: Nodes with maximum distance will be discarded. Tabu list consist of existing and new candidate value will be created.

Step 3: If the problem size is more significant than twice, then the prohibition period approaches to problem size 2. Iteration runs until the prohibition period reaches up to 1 .

Step 4: Nodes with least value will be stored in current solution. Further current solution list is now compared with the existing solutions.

\section{Algorithm D: Pseudocode for the Is Tabu function}

Input: Scurrt

Output: Tabu

1 Tabu $\leftarrow$ FALSE;

2 Scurrt feature $\leftarrow$ Retrieve Time Feature Last Used (Scurr feature);

3 if Scurrt feature $\geq$ Iteration curr - Prohibition Period then

4 Tabu $\leftarrow$ TRUE;

5 end

6 return Tabu; 
The steps involved in the Is Tabu function pseudocode have been described as follows.

Step 1: To find the Tabu list with reduced memory with the best move. List from the previous module will now be taken into consideration and compared whether best solution exist or not.

Step 2: The value is checked for the all the iterations i.e., current value and the previous feature.

Step 3: Maximum output value will be considered as the best one and stored for the further processes. If it results best deal, Tabu list will be created. Otherwise, steps will be repeated until we found the best one.

Step 4: If all conditions will be satisfied, final Tabu list will be created. Further results were generated and compared with the others.

Algorithm E: Pseudo code for the Modified Tabu Search Optimization (MTSO)

Algorithm Parameters used

$$
\begin{array}{ll}
\mathrm{n}=200,250,300 ; & \% \text { Number of nodes. } \\
\mathrm{l}=2 * \mathrm{n} ; & \% \text { Length of the network. } \\
\mathrm{w}=2 * \mathrm{n} ; & \% \text { Width of the network. } \\
\mathrm{R}=\mathrm{n} / 1.5 ; & \% \text { Radio range in meters. }
\end{array}
$$

Module 1: Generation of Parameters

$$
\begin{aligned}
& \text { node }_{x}=\operatorname{rand}([1, \mathrm{n}]) * 1 ; \\
& \text { node }_{y}=\operatorname{rand}([1, \mathrm{n}]) * \mathrm{w} ; \\
& \text { net }=\left[1: \mathrm{n} ; \boldsymbol{n o d e}_{x} ; \boldsymbol{n o d e}_{y}\right] ; \\
& \boldsymbol{o}(\boldsymbol{N e t})=\left[1: \mathrm{n} ; \boldsymbol{n o d e}_{\boldsymbol{x}} ; \boldsymbol{n o d e}_{\boldsymbol{y}}\right]
\end{aligned}
$$

Module 2: Find cost and memory optimization for each distance to zero $\left(\boldsymbol{X}_{\mathbf{1}}, \boldsymbol{Y}_{\mathbf{1}}=\mathbf{0}\right)$ and distance to $\mathrm{n}$-1 node for each node

$$
\begin{aligned}
& \text { for } \mathrm{i}=1: \mathrm{n}-1 \\
& X_{n}=\operatorname{node}_{x}(i, j) ; \\
& Y_{n}=\operatorname{node}_{y}(i, j) ; \\
& x_{\text {side }}=\operatorname{abs}\left(X_{2}-X_{1}\right) ; \\
& y_{\text {side }}=\operatorname{abs}\left(Y_{2}-Y_{2}\right) ; \\
& d=\operatorname{sqrt}\left(x_{\text {side }}^{2}+y_{\text {side }}^{2}\right) ; \\
& \text { end }
\end{aligned}
$$

Output :Cost, Memory $(1: n)=\operatorname{sum}($ dist $)$; 
Steps for Modified Tabu Search Optimization (MTSO)

1. Declare Algorithm Parameter.

2. Call Module 1 to generate parameters.

3. Call Module 2 to calculate the cost for each according to their distance and add them to further predict the net cost for each route iterationn.

maxIterations $=n$;

4. Repeat 2 to 3 for each node cost.

5. Repeat 6 to 8 for each node until the optimized node is found.

6. Create a list for each iteration:

Actions = TsCreateActionList(nVar);

7. A MTSO list for each action is now created at random position and their cost is calculated and best solution for the cost is found out:

$$
\begin{gathered}
T L 0=\operatorname{round}(0.05 * \text { nActions }) ; \\
T L=\operatorname{zeros}(\operatorname{size}(\text { Actions })) ; \\
\text { Sol.Position }=\operatorname{randperm}(\text { nVar }) ; \\
\text { Sol.Cost }=\text { CostFunction }(\text { Sol.Position }) ; \\
\text { BestSol }=\text { Sol; } \\
\text { BestCost }=\operatorname{zeros}(\text { maxiterations }, 1) ;
\end{gathered}
$$

8. For maximum iterationup to $n$ nodes, find the position and cost for each MTSO node:

$$
\begin{gathered}
\text { BestNewSol.Position = []; } \\
\text { BestNewSol.Cost }=\text { inf } ; \\
\text { BestAction }=0 ; \\
\text { for } k=1: \text { nActions } \\
\text { NewSol.Position }=\text { TsTourtion(Sol.Position, Actions }\{k\}) ; \\
\text { NewSol.Cost }=\text { CostFunction(NewSol.Position); }
\end{gathered}
$$


9. From the output of step 8, graphs have been plotted accordingly, and compared with the different models also.

\section{Modified Tabu Search (MTS) Simulated Outcomes}

In this study, simulated experimentation based on Modified Tabu Search (MTS) has been performed in dynamic scenario using MATLAB 2019a in order to determine the network shortest path and its allied cost. For experimentation, networks of 200, 250 and 300 node counts have been considered that has been positioned within $400 \mathrm{~m} \times 400 \mathrm{~m}, 500 \mathrm{~m} \times 500 \mathrm{~m}$ and $600 \mathrm{~m} \times 600 \mathrm{~m}$ area, respectively as depicted in Fig. 1 ( $\mathrm{a}, \mathrm{b}$ and $\mathrm{c})$. The radio range for 200, 250 and 300 nodes lie within 133.333m, 166.66m and 200m, respectively. Further, for each case first node acts as 'source' node which transferred data packets to the last node termed as 'terminal node'. Further, shortest path in a network has been computed by considering the path for which corresponding cost comes out to be the minimum.

a)

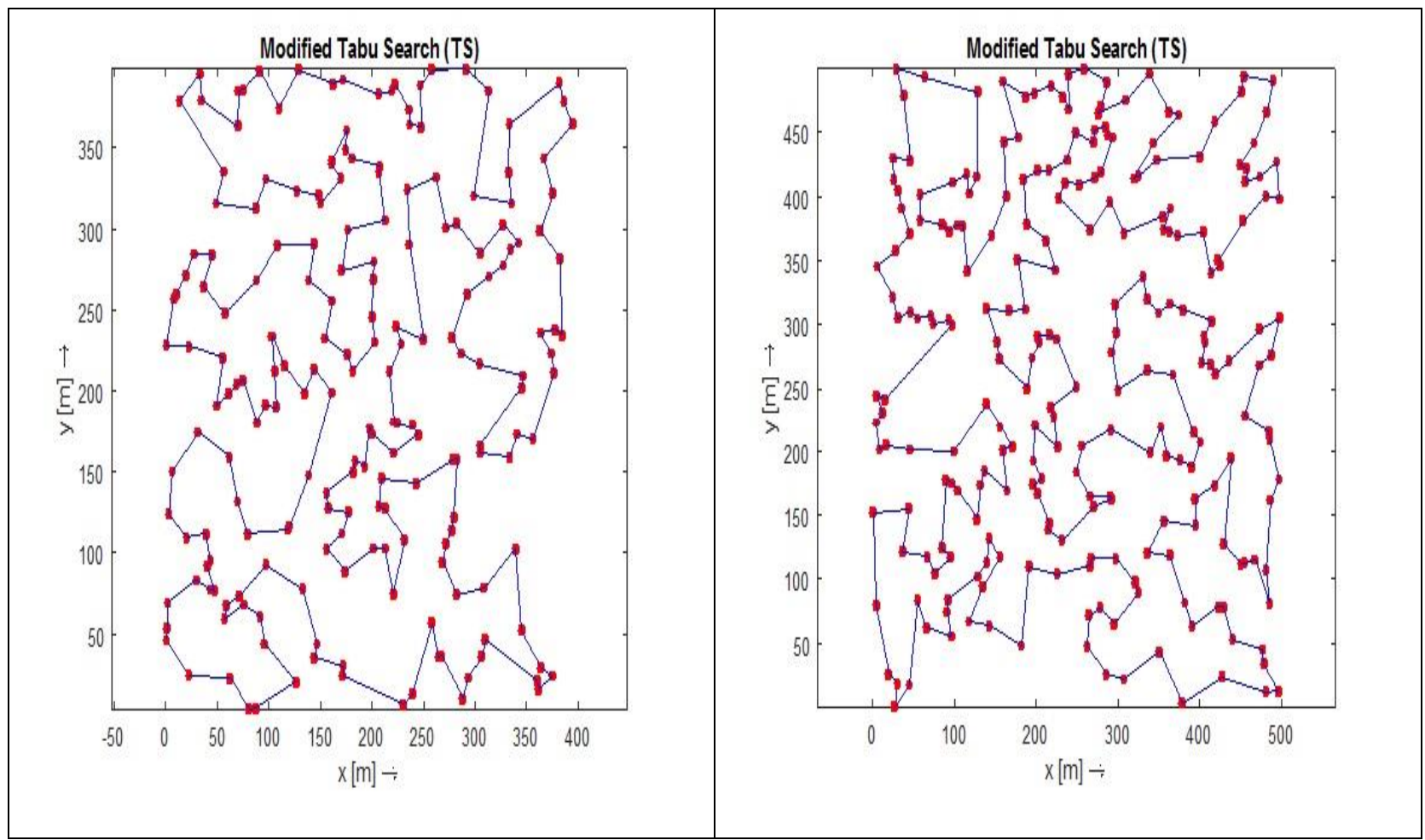


c)

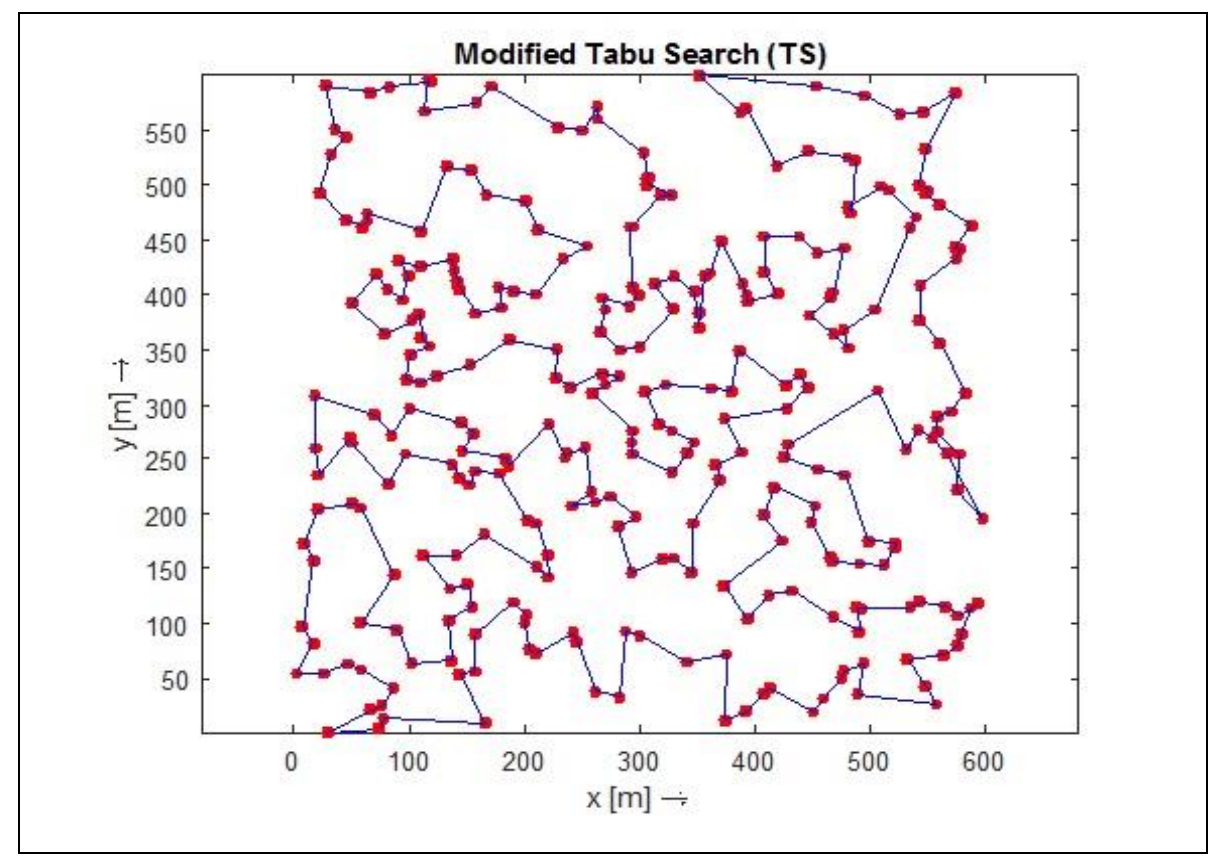

Fig. 1 Modified Tabu Search (MTS) approach for a) 200 nodes b) 250 nodes c) 300 nodes

\section{Comparative Study}

In this present study, different optimization techniques as discussed below have also been considered under similar working conditions for calculating the shortest path and its corresponding cost. Further, results obtained with different optimization approaches have been compared and analysed.

\subsection{Base Search Approach}

The Base approach has been regarded as a direct search strategy as it does not require derivatives to investigate a continuous domain. Base search approach has been related to procedures that allow limited progresses. Base search approach has the technique for modelling solutions globally across the entire search field, via a uniform distribution probability [16]. In this method, nodes have been connected to each other to build a mesh like framework. The implementation of Base network search approach for 200, 250 and 300 nodes has been highlighted in Fig.2 ( $a, b$ and $c$ ). 


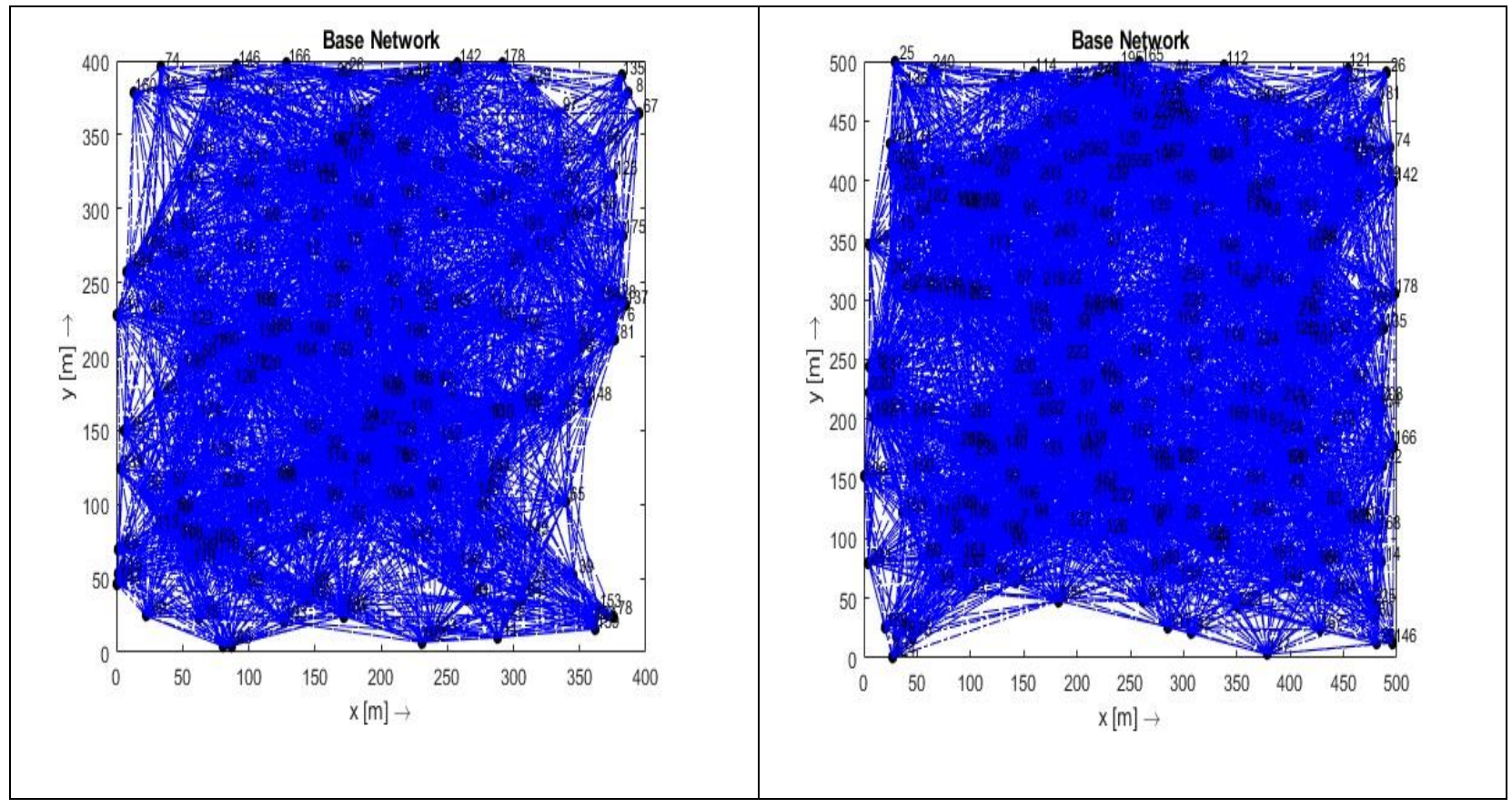

c)

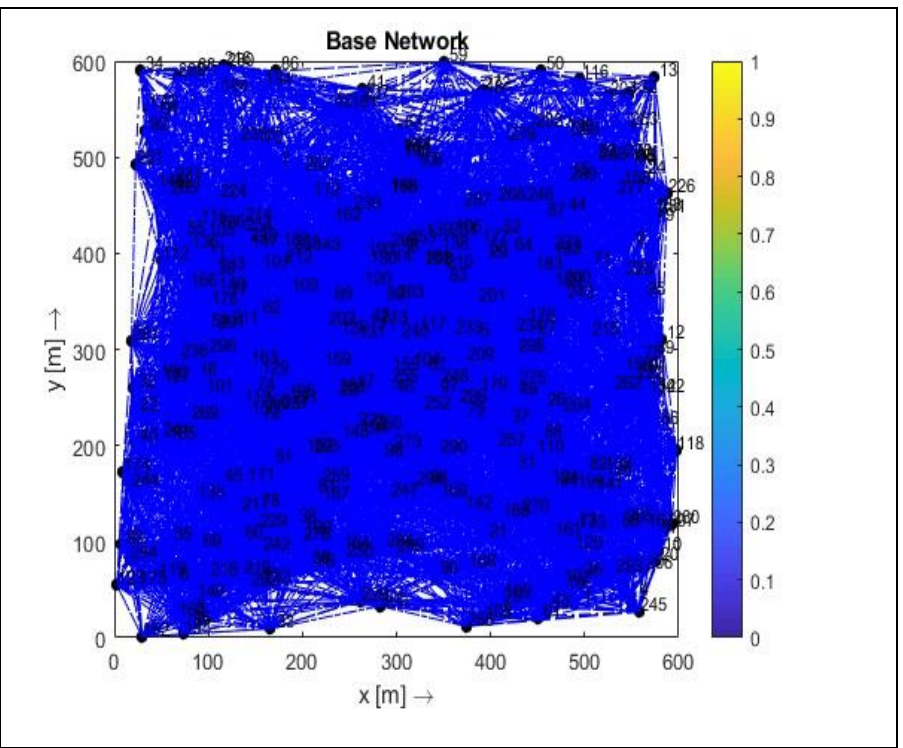

Fig. 2 Base search approach for a) 200 nodes b) 250 nodes c) 300 nodes

\subsection{Distance to Zero Search Approach}

The distance to zero search approach has been premeditated to address the confines of Base approach algorithm. The strategy for distance to zero search approach has to evaluate step size i.e., distance from zero required to reach the desired node in the search space to achieve 
global optimum. This has been accomplished by setting the distance from zero in ascending order to reach the desired node as depicted in Fig. 3 ( $a, b$ and c).

a)

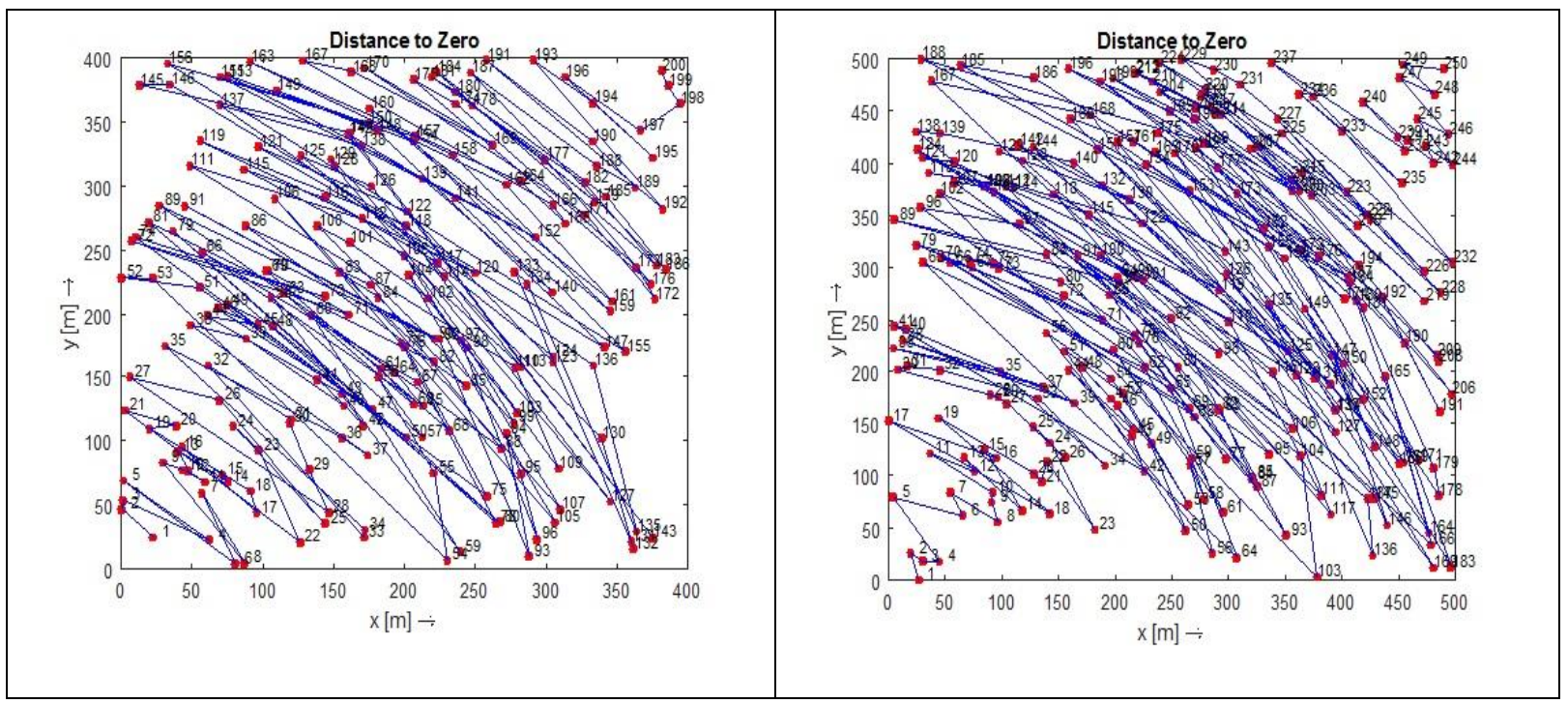

c)

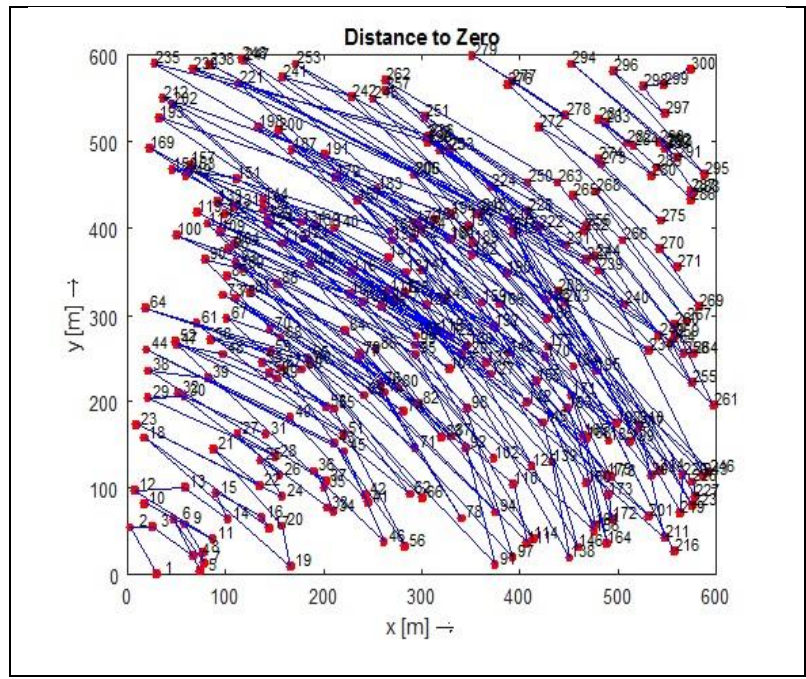

Fig. 3 Distance to zero search approach for a) 200 nodes b) 250 nodes c) 300 nodes

\subsection{Distance to Previous Node Approach}

The Distance to previous search algorithm has been a metaheuristic and general optimization approach algorithm that uses an embedded Local Search approach algorithm. This method is similar to the Tabu Search method algorithm, where the approximate cost functionality has been very close when simulation has been performed in MATLAB. In this approach distance has been calculated from last node to escape local optima and ascertain the global optima as 
shown in Fig. 4 ( $a, b$ and c). Distance to the previous search included iterative searches of more communities for a defined local optimum before an improvement is noticed, after which the search across increasing communities, recurrent. This approach may get aggravated on account of the following underlying principles:

- The local minimum for a neighboring node may not be a local-minima for another node.

- The global minimum has been a local minimum for all expected neighboring nodes.

- For many problem cases local minimum has been relatively similar to the global minimum.

a)

b)

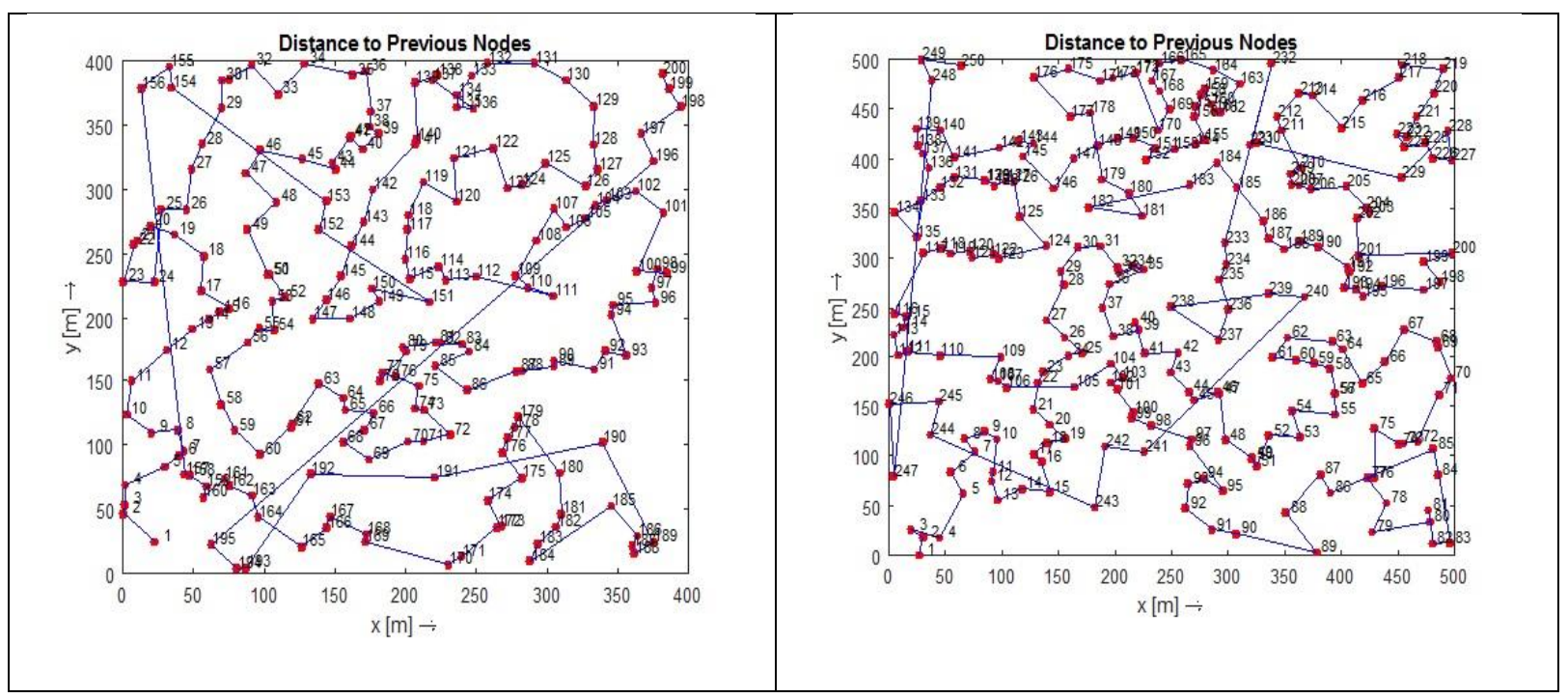

c)

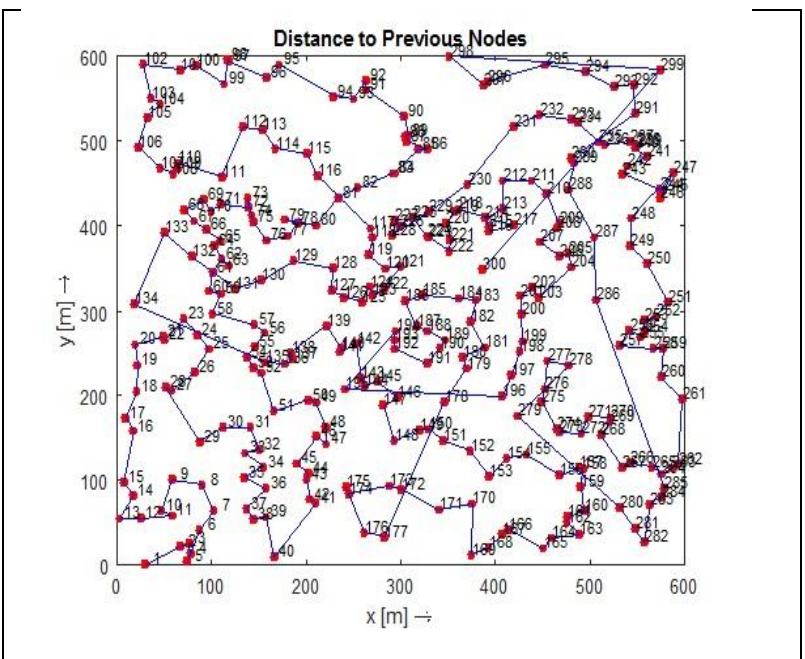


Fig. 4 Distance to previous node approach for a) 200 nodes b) 250 nodes c) 300 nodes

\subsection{Tabu Search Approach}

Tabu Search has been considered as an origin for large number of derivative techniques that present memory assemblies in Metaheuristics. The goal of Tabu Search approach is to force the embedded heuristic to recur at recently visited areas of the exploration space, identified as "cycling". The algorithm's approach has to maintain a short-term memory of the clear transformations of the latest moves inside the exploratory space and to prevent potential moves from not making those changes as seen in Fig. 5 (a, b and c).

a)

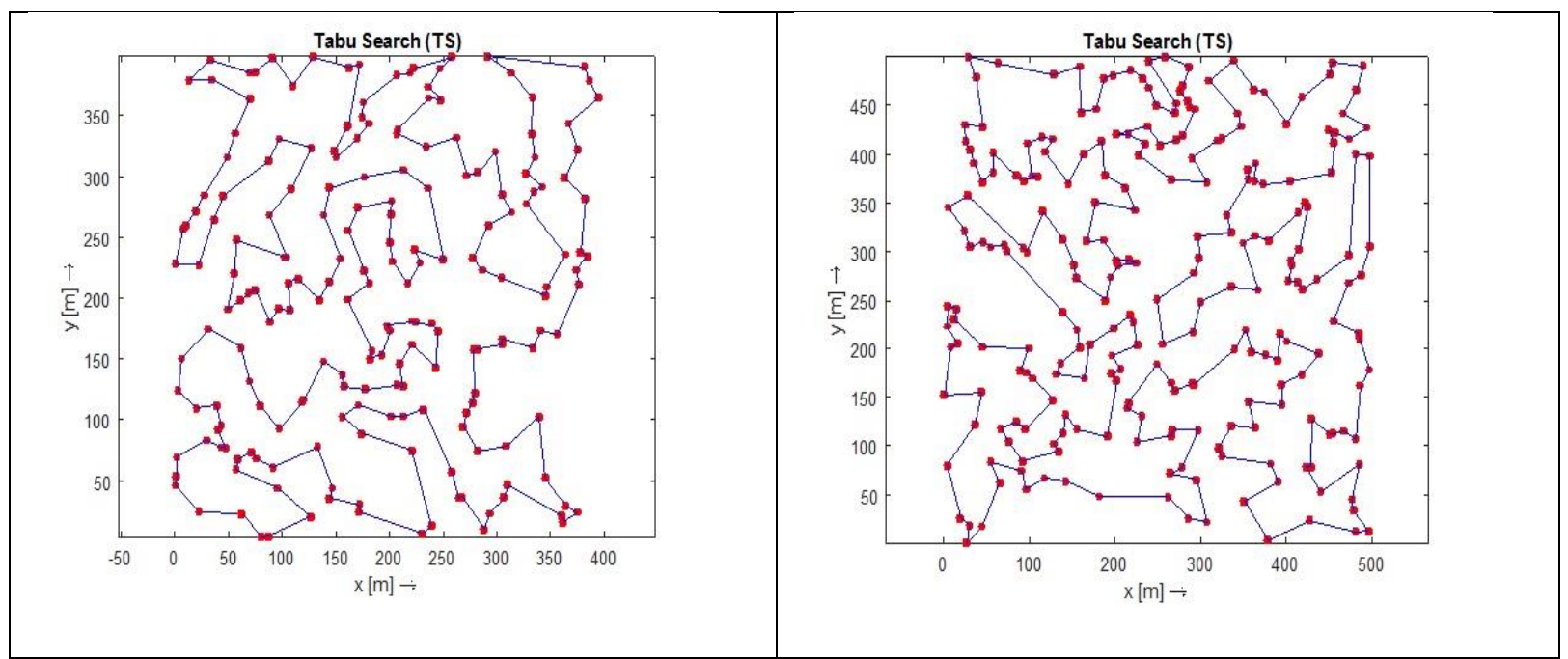

c)

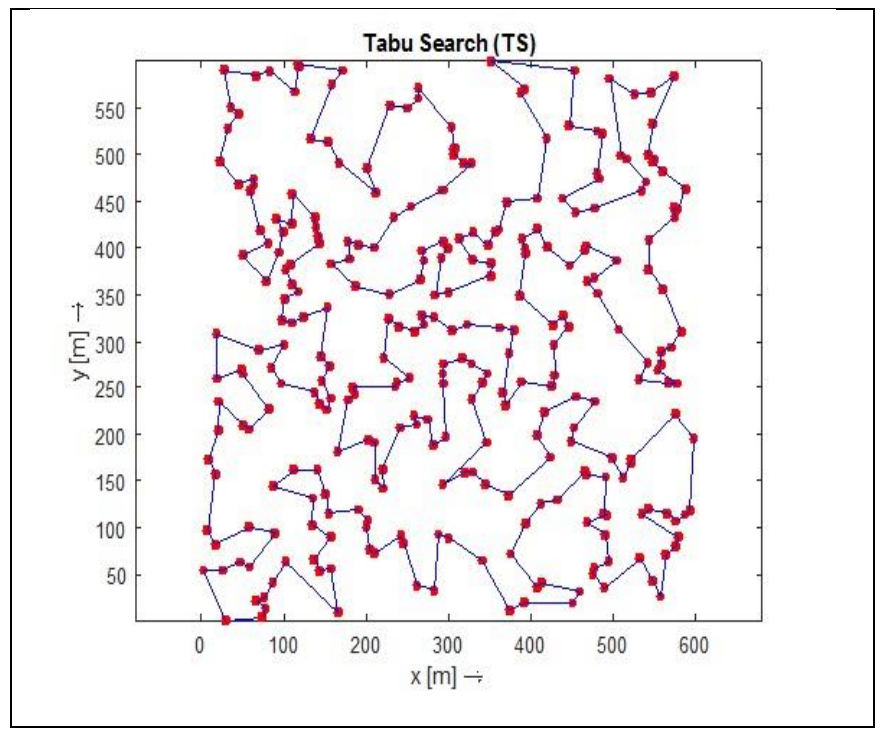


Fig. 5 Tabu Search approach for a) 200 nodes b) 250 nodes c) 300 nodes

\section{Results and Discussion}

Simulated results have been reported for above mentioned search algorithm approaches by considering 200, 250 and 300 node networks that have been depicted in Fig. 6 (a), (b) and (c), respectively. It is observed that in case of proposed Modified Tabu Search (MTS) algorithm, for a network size of 200 nodes, the cost associated with the shortest path is minimum, i.e., 4565.6024, while the cost in case of Base Search approach comes out to be 38510.5248 , i.e., maximum. Furthermore, it is observed that for greater node count i.e., 250 and 300, respectively Modified Tabu Search (MTS) approach outperformed all other optimization techniques in context with its cost effectiveness. Analogy of simulation results obtained via. Modified Tabu Search (MTS) algorithm with existing algorithms has been summed-up and has been depicted in Table 1 .

a)

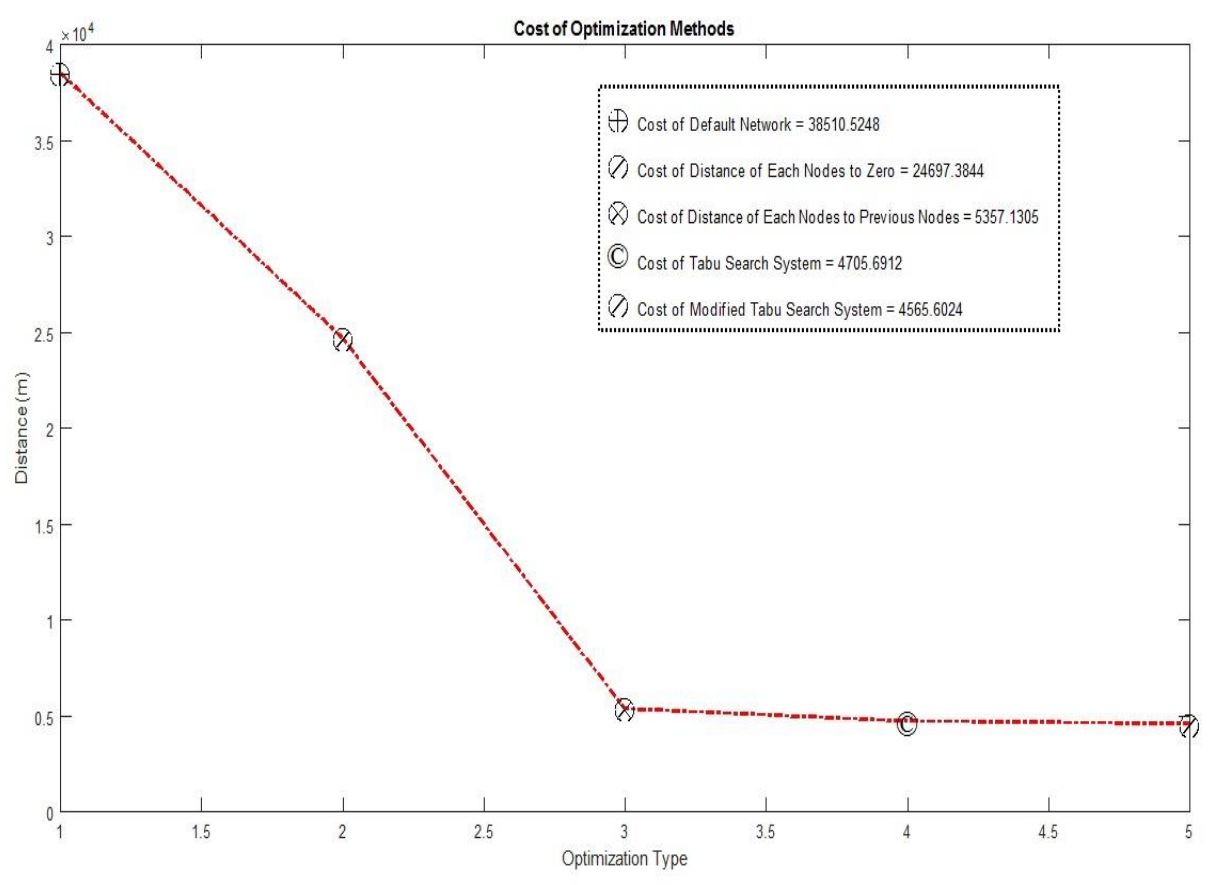


b)

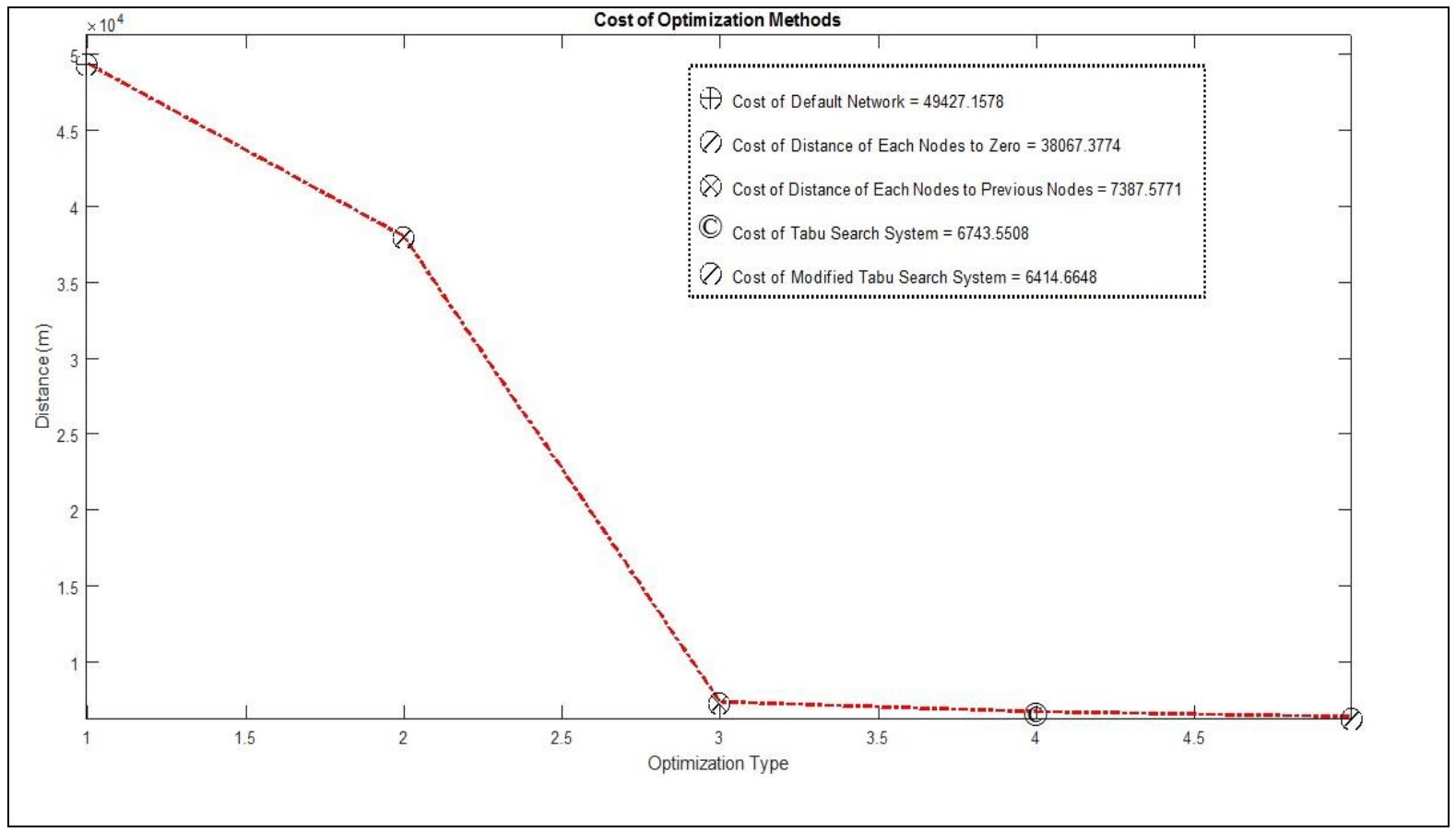

c)

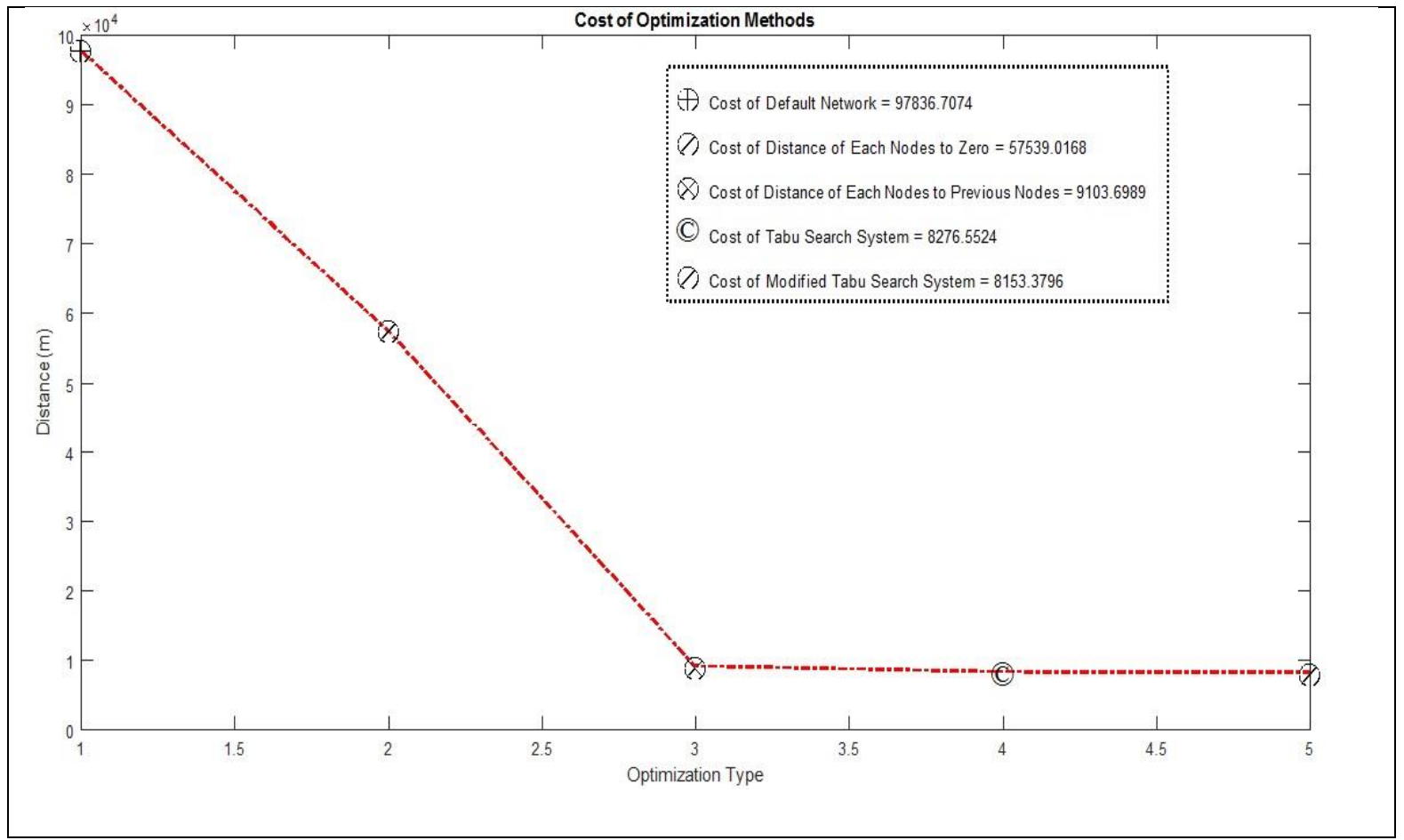

Fig. 6 Cost comparison simulated results for different optimization techniques a) 200 nodes b) 250 nodes c) 300 nodes 
Table 1 Analogy of simulation results with existing algorithms

\begin{tabular}{|c|c|c|c|}
\hline Routing algorithm & Node count (n) & $\begin{array}{c}\text { No. of links }=\mathrm{n} \\
(\mathrm{n}-1) / 2\end{array}$ & Path cost \\
\hline \multirow{3}{*}{ Base search } & 200 & 19,900 & 38510.5248 \\
\hline & 250 & 31,125 & 49427.1578 \\
\hline & 300 & 44,850 & 97836.7074 \\
\hline \multirow{3}{*}{ Distance to zero search } & 200 & 19,900 & 24697.3844 \\
\hline & 250 & 31,125 & 38067.3774 \\
\hline & 300 & 44,850 & 57539.0168 \\
\hline \multirow[t]{3}{*}{ Distance to previous node } & 200 & 19,900 & 5357.1305 \\
\hline & 250 & 31,125 & 7387.5771 \\
\hline & 300 & 44,850 & 9103.6989 \\
\hline \multirow{3}{*}{ Tabu search } & 200 & 19,900 & 4705.6912 \\
\hline & 250 & 31,125 & 6743.5508 \\
\hline & 300 & 44,850 & 8276.5524 \\
\hline \multirow{3}{*}{ Modified Tabu search } & 200 & 19,900 & 4565.6024 \\
\hline & 250 & 31,125 & 6414.6648 \\
\hline & 300 & 44,850 & 8153.3796 \\
\hline
\end{tabular}

\section{Conclusion}

In this piece of research work, the cost incurred in optimizing the shortest path for different network sizes has been computed for different routing algorithms and has been analyzed. From simulation results computed for all network sizes considered in the study, it is concluded that Modified Tabu Search (MTS) algorithm outperformed all other approaches as it involved minimum cost for the shortest path, thereby, proving to be a dominant algorithmic approach being functional with immense solution to numerous intricate combinatorial 
tribulations while all other meta-heuristic techniques need both the scope and complexity of their incisive method to be obtained, an issue of complexity is not applicable for MTS. Therefore, MTS can be considered to be able to solve the combinatorial optimization problems quite efficiently and effectively in an IOT based network.

\section{Acknowledgment}

The authors would like to acknowledge the Maharishi Markandeshwar (Deemed to be) University, Mullana, India, for providing the facilities required to carry out this research work.

Author Contributions Anukriti Sharma: Conceptualization, Data Curation, Writing Original Draft, Writing - Review \& Editing (Corresponding Author).

Sharad Sharma: Review \& Editing, Supervision (Co - Author).

Dushyant Gupta: Review \& Editing, Supervision (Co - Author).

Data Availability No supplementary data and material are needed for this research work

\section{Compliance with Ethical Standards}

The authors ensure that accepted principles of ethical and professional conduct have been followed during this research work.

Conflict of Interest None.

Consent to Participate Not applicable

Consent for Publication The authors give full consent for publication of this research work 


\section{References}

1. Glover, F., Tabu search — part II. ORSA Journal on computing, 1990. 2(1): p. 4-32.

2. Glover, F., Tabu search and adaptive memory programming - advances, applications and challenges, in Interfaces in computer science and operations research. 1997, Springer. p. 1-75.

3. Youssef, H., S.M. Sait, and H. Adiche, Evolutionary algorithms, simulated annealing and tabu search: a comparative study. Engineering Applications of Artificial Intelligence, 2001. 14(2): p. 167-181.

4. $\quad$ Reeves, C.R., Modern heuristic techniques for combinatorial problems. 1993: John Wiley \& Sons, Inc.

5. de Werra, D. and A. Hertz, Tabu search techniques. Operations-Research-Spektrum, 1989. 11(3): p. 131-141.

6. Fang, Y., et al. Tabu search algorithm based on insertion method. in International Conference on Neural Networks and Signal Processing, 2003. Proceedings of the 2003. 2003. IEEE.

7. Anukriti Sharma, S.S., Advances and Challenges in the IOT Routing Protocols: A Comprehensive Review. International Journal of Advanced Science and Technology, 2020. 29(04): p. 9350 - 9371.

8. Mathlouthi, I., M. Gendreau, and J.-Y. Potvin, A metaheuristic based on Tabu search for solving a technician routing and scheduling problem. Computers \& Operations Research, 2020: p. 105079.

9. Hertz, A. and T. Ridremont, A tabu search for the design of capacitated rooted survivable planar networks. Journal of Heuristics, 2020: p. 1-22.

10. Chen, M., et al., A Tabu search algorithm with controlled randomization for constructing feasible university course timetables. Computers \& Operations Research, 2020: p. 105007.

11. Sharma, S., S. Kumar, and B. Singh, Routing in wireless mesh networks: Three new nature inspired approaches. Wireless Personal Communications, 2015. 83(4): p. 3157-3179.

12. Sharma, S., S. Kumar, and B. Singh, Hybrid intelligent routing in wireless mesh networks: soft computing based approaches. International Journal of Intelligent Systems and Applications (IJISA), 2014. 6(1): p. 45-57.

13. Sharma, S., S. Kumar, and B. Singh, AntMeshNet: An ant colony optimization based routing approach to wireless mesh networks. International Journal of Applied Metaheuristic Computing (IJAMC), 2014. 5(1): p. 20-45.

14. Kumar, S., B. Singh, and S. Sharma, Soft computing framework for routing in wireless mesh networks: an integrated cost function approach. arXiv preprint arXiv:1307.3011, 2013.

15. Battiti, R. and G. Tecchiolli, The reactive tabu search. ORSA journal on computing, 1994. 6(2): p. 126-140.

16. Andradóttir, S., A global search method for discrete stochastic optimization. SIAM Journal on Optimization, 1996. 6(2): p. 513-530. 


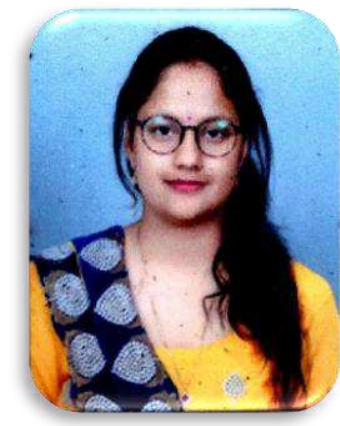

Anukriti Sharma received her B. Tech degree in Electronics and Communication Engineering from Kurukshetra University, Kurukshetra, India in 2011 and M. Tech in Electronics and Communication Engineering from Kurukshetra University, Kurukshetra, India in 2014. She is currently a Ph.D. candidate of Electronics and Communication Engineering in Maharishi Markandeshwar University, Mullana, India. Her research interests are Wireless Communication, Internet of Things and Soft Computing etc.

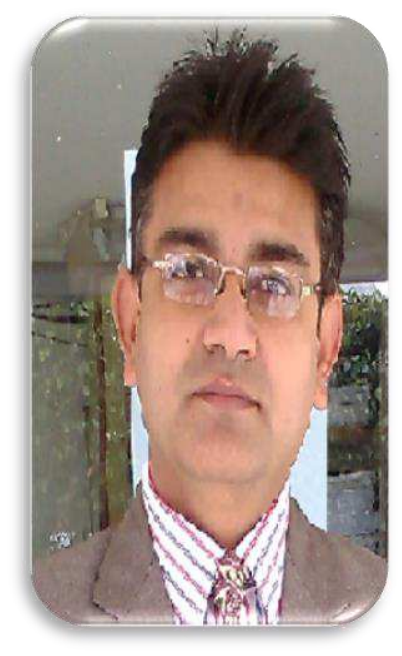

Dr. Sharad Sharma has a vast experience of more than 20 years of teaching and administrative work. He has received his B. Tech degree in Electronics Engineering from Nagpur University, Nagpur, India, M. Tech in Electronics and Communication Engineering from Thapar Institute of Engineering and Technology, Patiala, India and Ph.D. from National Institute of Technology, Kurukshetra, India. He has a teaching experience of 20 years. He has conducted many workshops on Soft Computing and its applications in engineering, Wireless Networks, Simulators etc. He has a keen interest in teaching and implementing the latest techniques related to wireless and mobile communications. He has opened up a student chapter of IEEE as Branch Counsellor. His research interests are routing protocol design, performance evaluation and optimization for wireless mesh networks using nature inspired computing, Internet of Things and Space Communication etc. He is member of various professional bodies such as IEEE, IACSIT and Internet Society.

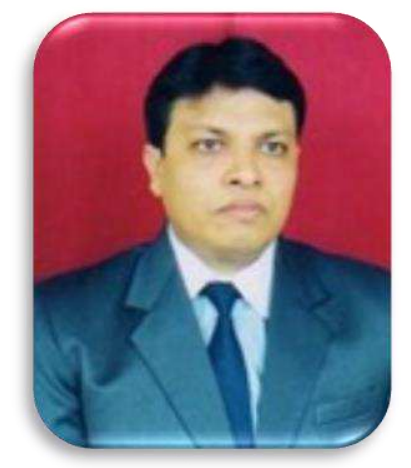

Dr. Dushyant Gupta presently working as an Associate Professor in the Electronics Department, Institute of Hons. \& Evening Studies, Kurukshetra University, Kurukshetra, India. Prior to this post, He has been working as a Professor in the ECE Department, G.J. University of Science and Technology, Hisar, India. He has a teaching experience of 28 years. He has published 40 paper in various journals/ conferences of national and international repute. He is associated with several journals as a reviewer and co-editor. He is member of various professional bodies. 